\title{
Influence of work zone signage on driver speeding behavior
}

\author{
Snehanshu Banerjee $^{1} \cdot$ Mansoureh Jeihani $^{1} \cdot$ Nashid K. Khadem $^{1}$
}

Received: 6 November 2018/Revised: 7 January 2019/Accepted: 11 January 2019/Published online: 1 February 2019

(C) The Author(s) 2019

\begin{abstract}
Speeding in a work zone needs to be tackled effectively to enhance work zone safety. This study evaluates the influence of three different work zone signsspeed photo enforced signs, dynamic speed display signs and reduced speed limit signs-on driver speeding behavior using a medium-fidelity driving simulator. The speed photo enforced and reduced speed limit signs were placed at intervals before the start of the work zone and in the work zone. A virtual work zone network was built to replicate the Baltimore-Washington Parkway (MD-295). A total of 66 participants from different socioeconomic backgrounds participated in 264 driving simulation sessions. The speeds were measured over four phases: initial speed area, sign visible area, sign readable area and post sign area. An analysis of variance and post hoc analysis showed that the speed photo enforced sign was the most effective of the three signs. The second speed photo enforced sign within the work zone was more effective at reducing speed than the sign before the start of the work zone, especially in the post sign area. It was observed that female participants tend to slow down more when encountering a speed photo enforcement sign.
\end{abstract}

Mansoureh Jeihani

mansoureh.jeihani@morgan.edu

Snehanshu Banerjee

snban1@morgan.edu

Nashid K. Khadem

nakha3@morgan.edu

1 Department of Transportation and Urban Infrastructure Studies, Morgan State University, 1700 E. Cold Spring Lane, Baltimore, MD 21251, USA
Keywords Work zone - Driving simulator - Speed study · Speed photo enforced sign · Dynamic speed display sign . Reduced speed limit sign

\section{Introduction}

A significant threat is posed by speeding in work zones to drivers as well as workers. The National Highway Traffic Safety Administration (NHTSA) reported that in 2017, accidents within designated work zones included around 710 fatal accidents involving 799 fatalities [1]. Every year, more than 100 crew members die in work zone-related crashes [2]. The total cost of work zone-related crashes is in the billions of dollars and is expected to grow due to increased traffic [3]. These numbers can be reduced by innovations in work zone signage. However, implementation of such interventions before proper validation can undermine rather than enhance safety and may lead to potentially fatal outcomes [4].

The majority of the research focuses on evaluating the influence of a single sign on speeding behavior [2, 5-8]. Thus, from these studies, the effectiveness of different signs cannot be truly compared since they are not subjected to the same conditions and study environment. This study evaluates the effects of three different signs-speed photo enforced signs, dynamic speed display signs (DSDS) and reduced speed limit signs or R2-1 in conjunction with G20$5 \mathrm{aP}$, according to the Manual on Uniform Traffic Control Devices (MUTCD) naming convention [9]—on speeding behavior in a high-speed work zone. Speed photo enforced signs and reduced speed limit signs are placed before the start of the work zone and in the work zone to test the effect on speeding. Results from this study can be used by safety experts to deploy the most effective signage to 
compel drivers to slow down in a work zone, thereby safeguarding both the driver and the work zone crews. For enhanced traffic safety, studying driver behavior is fundamentally significant [4]. Diverse factors impact the speed of vehicles traveling through a work zone, such as road geometry, warning signs and traffic control devices, speed displays and law enforcement [10]. In different road conditions, drivers alter their vehicle speeds in response to traffic control devices and roadway geometry [5]. The following is a summary of prior research on speeding behavior using different signs.

Different display signs are used to influence driver speeding behavior in a work zone. Dynamic speed display signs (DSDS), variable speed limit (VSL), portable changeable message signs (PCMS), reduced speed limit signs and speed photo enforced signs are the most commonly used signs $[6,7,11]$. DSDS present a means to enhance road safety, and their application has been primarily in work zones. The effectiveness of DSDS has been studied in the past by several researchers [6,7]. The effectiveness of DSDS and photo radar were compared, and both the devices reduced vehicle speeds by $4-5 \mathrm{mph}$ and curbed the number of vehicles that were traveling $10 \mathrm{mph}$ or more over the prescribed speed limit of $25 \mathrm{mph}$ on single-lane neighborhood roads [11]. DSDS and VSL sign placement in work zones was found to be useful in other studies, too, in which a driving simulator was used [7]. Signs containing messages like "SLOW DOWN" combined with VSL signs actually led drivers to reduce speeds [7]. It was also found that the message "SLOW DOWN" reduced the speed by $2 \mathrm{mph}$ more than VSL alone when posted work zone speed limit was $45 \mathrm{mph}$. When DSDS were active, the speed was reduced by an average of $1.4 \mathrm{mph}$ when the posted speed limit was $30 \mathrm{mph}$ on two-way single-carriageway roads [12] and greater than $10 \mathrm{mph}$ in a high-speed bridge reconstruction work zone with a speed limit of $55 \mathrm{mph}$ [6]. It was also observed that DSDS were effective for short distances only and drivers increased their speed after they passed the sign [8]. Moreover, studies conclude that the effectiveness of DSDS increased when they were combined with another device for speed control like a speed camera.

A speed photo enforcement sign is another intervention that is implemented to influence driver behavior in work zones. Speed cameras and law enforcement presence play effective roles in the reduction in average speed and enhance work zone speed limit compliance $[5,13,14]$. The presence of photo-radar enforcement and speed display boards helped reduce vehicle speed by $4.3-5 \mathrm{mph}$ where the work zone posted speed limit was $55 \mathrm{mph}$ for cars and trucks, resulting in a $16 \%$ reduction in the number of speeding vehicles [15]. The presence of speed cameras led average free-flowing speed to decrease by $4.2-7.9 \mathrm{mph}$ for cars and 3.4-6.8 mph for trucks, when two lanes were open to traffic and posted speed limit was $55 \mathrm{mph}$ [5]. This implementation significantly reduced the number of cars and trucks exceeding the speed limit in the work zone. Another study found that speed photo-radar enforcement reduced the car speeds by $2-3.8 \mathrm{mph}$ and trucks by $0.8-5.3 \mathrm{mph}$ on average, where the posted speed limit was $55 \mathrm{mph}$ [16]. Some researchers found that the drivers believe that speed should not be reduced when work is not in progress in the work zone or when the work zone is not adjacent to the traveled way, and they reduced speeds only when workers are present in the work zone [17, 18].

PCMS are part of intelligent transportation systems. They are used as temporary traffic control (TTC) devices. These are also used in the advance warning area of a work zone to provide real-time information to drivers. These signs are also effective in work zones and help reduce vehicle speeds $[19,20]$. Studies found that concise and clearly worded messages have the most impact in influencing speed. Messages with both speed and time limits were found to be the most effective on drivers in reducing overall speed [19]. This speed reduction was a combined effort of four PCMS used in a work zone. In another study, driver behavior was investigated through sequential PCMS [20]. It was found that drivers slow down more when a specific speed limit is shown on PCMS. Sometimes drivers do not follow the messages on the PCMS as they doubt the reliability of the signs [21]. The drivers ignore the signs and do not slow down if the location of the sign is far from the work zone [13].

Reduced speed limit signs are another intervention that has shown varying degrees of effectiveness in past studies [22]. However, the effectiveness of reduced speed limit signs may be dependent on sight distance, geometry of the road and posted speed limits, while in some cases the effectiveness may depend on the work zone location [22]. Reduced speed limit signs for extended work zones that have no ongoing activity are ineffective and make drivers skeptical of additional reduced speed limit signs posted in other work zones [23]. Another study found that reduced speed limit signs have very little effect on speed reduction, and the presence of law enforcement officers was more effective when compared to such signs [13].

Numerous studies have been conducted to evaluate driver behavior under various circumstances using a driving simulator [24-31]. Some studies investigated the impact of work zone barriers (cone pylons, concrete jersey barriers and metal barriers) on driver behavior [24], while some analyze drivers' behavior under different climatic conditions like foggy conditions and reduced visibility $[25,29,30]$. Most of the simulator studies investigated the impact of different kind of message signs on driver's route choice and compliance behavior, diversion of traffic, speed 
patterns, sign effectiveness and safety of traffic [20, 26-29, 31]. This study focuses on work zone safety and evaluates the influence of three different work zone signs-speed photo enforced signs, DSDS and reduced speed limit signs-on driver speeding behavior using a medium-fidelity driving simulator.

\section{Methodology}

A medium-fidelity driving simulator (Fig. 1) at the Safety and Behavioral Analysis (SABA) Center at Morgan State University was used to investigate the impact of work zone signs on speeding behavior in this study. A virtual work zone environment on the Baltimore-Washington Parkway

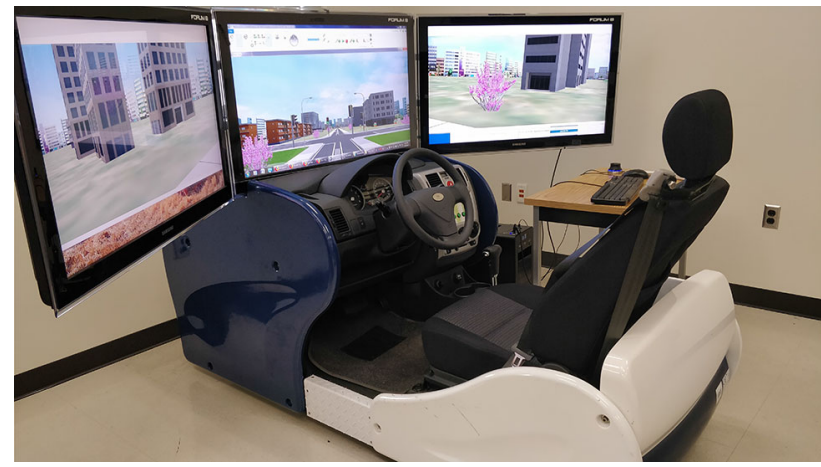

Fig. 1 Driving simulator at the SABA Center, Morgan State University
(MD-295) was created using the software VR-Design Studio developed by FORUM8 Co. [32]. Data such as lane changing, acceleration, braking, steering control and speed were recorded in real time by the driving simulator software.

MD-295 connects Washington, DC, to Baltimore, Maryland, and has a speed limit of $55 \mathrm{mph}$. The simulation was carried out over approximately 9500 feet (1.8 miles), consisting of three 12-foot lanes and a reduced speed limit of $35 \mathrm{mph}$ within the work zone. Only one lane was open, while the other two were closed for construction. Warning signs were used in the advance warning area. Daytime driving conditions with no significant weather impacts were chosen. A schematic of the work zone area is shown in Fig. 2.

Four phases of driver speed were tested as they approached and passed the signs. When the participant is at or above the posted speed limit, the initial speed area is an arbitrary distance of 375 feet before the start of the next phase. The visible area and readable area distances were measured by three independent people, i.e., the distances at which the sign became visible and legible to them. These distances were averaged and used for calculations. The post sign area distance is another arbitrary distance of 250 feet to assess the impact on speed right after passing the sign. The different phases along with the signs used are shown in Fig. 3.

The control scenario displays all the advance warning signs and posted speed limits used in all subsequent scenarios. Speed photo enforced signs and reduced speed limit

(a)

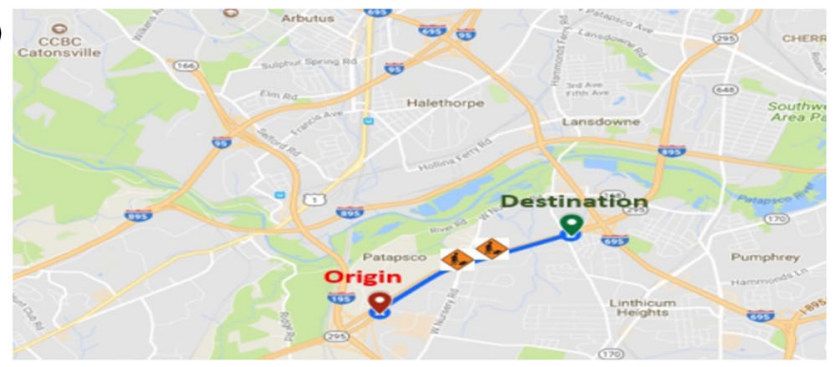

(b)

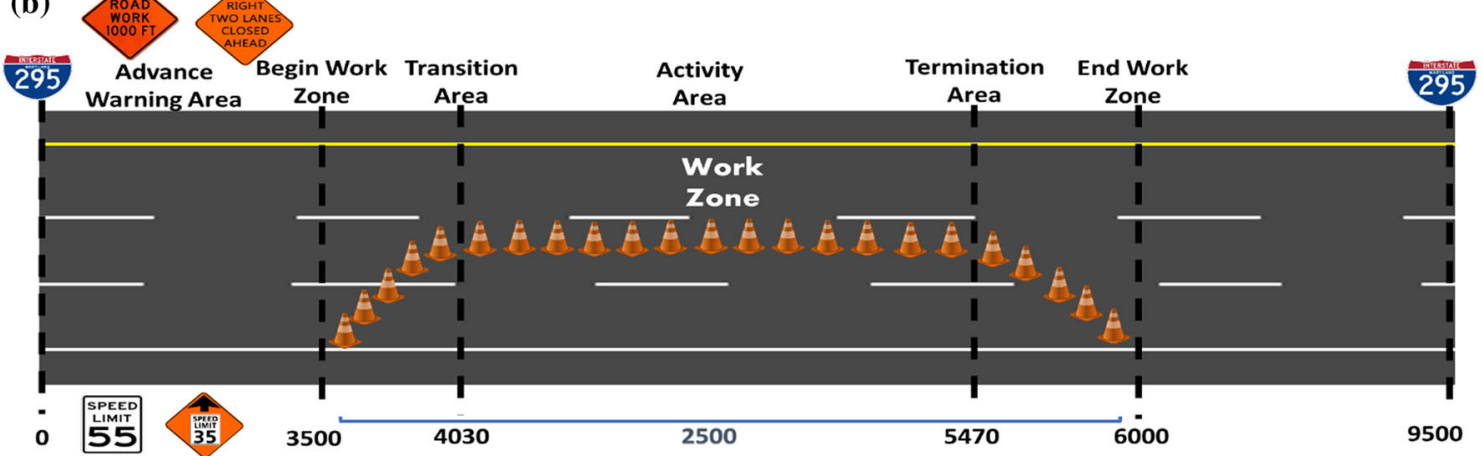

Fig. 2 The study corridor (all dimensions are in feet and are not to scale). a Work zone on MD-295, Baltimore, b work zone schematic (control scenario) 

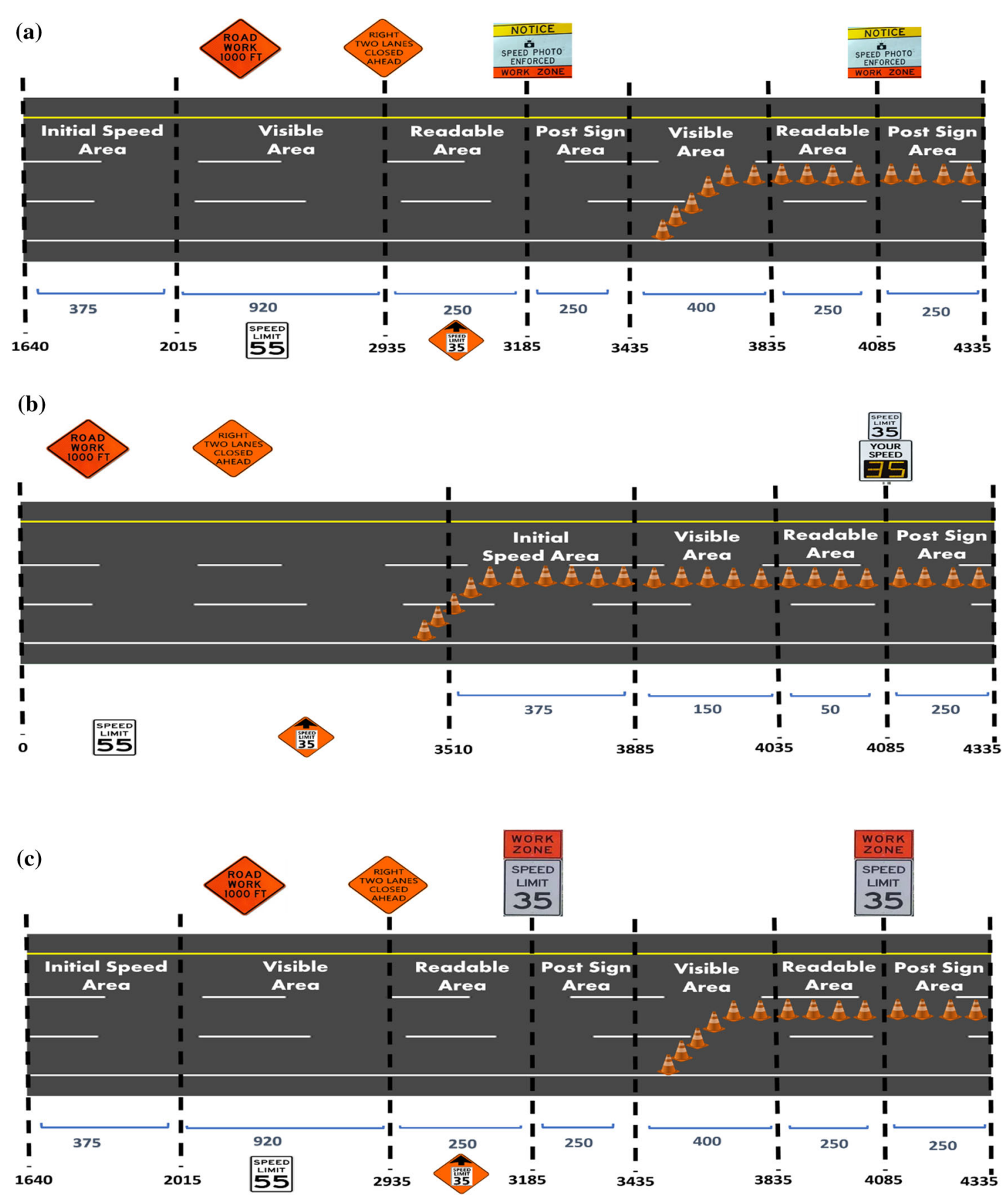

Fig. 3 Work zone signs tested in this study (all dimensions are in feet and are not to scale). a Work zone with speed photo enforced signs, b work zone with DSDS, $\mathbf{c}$ work zone with reduced speed limit signs

signs are placed before the start of the work zone and in the work zone to test the impact of placing consecutive signs on speeding. The visible and readable area distances can differ depending upon various factors such as road geometry, size of the sign and traffic ahead, among other things. Although comparing two speed photo enforced signs and two reduced speed limit signs to a single DSDS seems imbalanced, in the real world two DSDS in a single work zone are rarely seen. The study area is a straight road and thus the placement of signs in the work zone was not considered as an issue. Although researchers consulted the MUTCD for the minimum standard distances for visibility, sign location and sign size, the distances and sign size could not be adhered to, as cognition in the simulator is different from the real world. Since vision and perception can differ for every participant, three random volunteer 
participants in the age groups of 18-25 (male), 36-45 (male) and 56-65 (female) were chosen and their perception distances for the different signs were averaged and then considered as the standard distances for a particular sign and used for analysis. The traffic for this study was set at level of service (LOS) B as defined by the highway capacity manual (HCM), i.e., light traffic, so that vehicles do not slow down on account of traffic, and the actual influence of work zone signs can be tested. Two photo enforced signs as well as reduced speed limit signs were tested, one before the start of the work zone and the other inside the work zone.

Before the start of the study, the steering wheel and pedals of the driving simulator were calibrated. The participants in this study were asked to drive four different scenarios, with each scenario incorporating different signs and one scenario as the control scenario with only advance warning signs. The control scenario was always used as the first scenario to ensure participants do not know when to expect signage in the work zone and drive as they would in real life.

\section{Survey questionnaires}

All participants were invited to fill out a socio-demographic survey prior to the start of the simulation session. The survey was designed to capture essential information about the participants with regard to gender, age, level of education and annual household income. This socio-demographic information was used to investigate any correlation between speeding behavior and the sociodemographic characteristics of the participants.

After the completion of the four simulation sessions, participants were asked to fill out a post-simulation survey, in which they were asked about the level and type of discomfort, if any, experienced during the simulation session and their experience using the driving simulator.

\section{Study data}

Prior approval from the institutional review board (IRB) was received before participants were recruited for the study. Participants were invited at random, by word of mouth, through the distribution of fliers across Morgan State University (MSU), Towson University, Baltimore County and Baltimore City and by placing ads online.

They were paid $\$ 15 / \mathrm{h}$ for their participation. They were informed that they would be penalized for rash driving and getting involved in crashes to ensure driving realism.

A total of 66 individuals participated over 264 simulation sessions, which involved a balance of males and
Table 1 Socio-demographic characteristics of the participants

\begin{tabular}{llr}
\hline Variables & Description & $(\%)$ \\
\hline Gender & Male & 55 \\
Age & Female & 45 \\
& $18-25$ & 33 \\
& $26-35$ & 39 \\
& $36-45$ & 11 \\
Familiarity with study area & $46-55$ & 10 \\
& $56-65$ & 7 \\
& Yes & 57 \\
Education & Nomewhat & 29 \\
& Ho & 14 \\
& Associate degree & 14 \\
& Undergraduate student & 15 \\
& Undergraduate degree completed & 36 \\
Household income & Postgraduate student & 15 \\
& Postgraduate degree completed & 9 \\
& $<\$ 20,000$ & 42 \\
& $\$ 20,000-\$ 29,999$ & 15 \\
& $\$ 30,000-\$ 49,999$ & 23 \\
& $>\$ 50,000$ & 20 \\
\hline
\end{tabular}

females. The control scenario was always tested first followed by scenario involving reduced speed limit sign, DSDS and finally photo enforced sign. Table 1 presents the descriptive statistics of the participants. The participants were given the opportunity to become familiar with the driving simulator before the start of the simulation session.

\section{Analysis and discussion}

An ANOVA was conducted to identify the differences in mean speed across the various phases for each of the signs tested in the work zone. The mean speeds at different phases and analysis of variance (ANOVA) significance for each sign are given in Table 2 .

Table 2 shows that there is a statistically significant difference in mean speeds across the different phases in the presence of photo enforced work zone signs and DSDS. The reduced speed limit 35 sign does not seem to cause a statistically significant change in speed. The mean speeds in the work zone in the absence of signage are approximately equal to that of the highway speed limit of $55 \mathrm{mph}$ at MD-295. Table 3 displays the comparison of mean speed by phase, in the presence of work zone photo enforced signs.

Table 3 shows that the initial photo enforced work zone sign outside the work zone did not have as much effect as the next photo enforced sign (sign II) inside the work zone. 
Table 2 Descriptive statistics and ANOVA of different phases by sign

\begin{tabular}{lcclll}
\hline Work zone photo enforced signs & & & & \\
\hline Phases & $\begin{array}{l}\text { Mean } \\
(\mathrm{mph})\end{array}$ & \multicolumn{1}{l}{ SD } & SE & $F$ & Sig. \\
& & & & \\
\hline Initial speed area & 49.508 & 4.789 & 0.599 & 16.723 & $0.000^{*}$ \\
Visible area & 50.221 & 8.731 & 1.091 & & \\
Readable area & 51.420 & 9.615 & 1.202 & & \\
Post sign area & 49.947 & 9.657 & 1.207 & & \\
Visible area (sign & 46.245 & 9.817 & 1.227 & & \\
$\quad$ II) & & & & & \\
Readable area (sign & 41.769 & 11.228 & 1.404 & & \\
$\quad$ II) & & & & & \\
Post sign area (sign & 38.928 & 10.399 & 1.300 & & \\
$\quad$ II) & & & & & \\
Dynamic speed display sign (DSDS) & & & & \\
Initial speed area & 56.158 & 8.908 & 1.105 & 6.841 & $0.000^{*}$ \\
Visible area & 53.214 & 10.315 & 1.279 & & \\
Readable area & 49.993 & 11.564 & 1.434 & & \\
Post sign area & 48.233 & 12.185 & 1.511 & & \\
Reduced speed limit & (SL) 35 signs & & & \\
Initial speed area & 55.668 & 9.407 & 1.167 & 1.926 & 0.075 \\
Visible area & 53.153 & 10.893 & 1.351 & & \\
Readable area & 53.083 & 10.033 & 1.244 & & \\
Post sign area & 52.360 & 10.128 & 1.256 & & \\
Visible area (sign & 51.698 & 10.395 & 1.289 & & \\
II) & & & & & \\
Readable area (sign & 50.970 & 10.643 & 1.320 & & \\
II) & & & & & \\
Post sign area (sign & 50.211 & 10.824 & 1.343 & & \\
$\quad$ II) & & & & & \\
No signs (control scenario) & & & \\
Work zone & 55.883 & 9.586 & 1.180 & - \\
\hline
\end{tabular}

*Significant at $95 \%$ confidence interval

The participants reduced their speed after the first sign they encountered and continued to reduce their speed as they encountered the second sign. This shows that having consecutive work zone photo enforced signs at suitable intervals (900 feet in this study) could possibly lead participants to lower their speeds in the work zone. The results also show that the decrease in speed takes place when the sign becomes readable and after passing the sign. The total reduction in speed from the initial speed area to after passing the work zone photo enforced sign was approximately $11 \mathrm{mph}$.

Table 4 shows the comparison of mean speed by phase in the presence of DSDS. The participants reduced their speeds gradually once the DSDS became visible, readable and after passing the sign as well. The total reduction in speed from the initial speed area to after passing the DSDS was approximately $8 \mathrm{mph}$.
Table 3 Tukey's post hoc analysis-work zone photo enforced signs

\begin{tabular}{|c|c|c|}
\hline Phase comparison & Mean difference (I-J) & Sig. \\
\hline \multicolumn{3}{|l|}{ Initial speed area } \\
\hline Visible area & -0.713 & 1.000 \\
\hline Readable area & -1.912 & 0.911 \\
\hline Post sign area & -0.439 & 1.000 \\
\hline Visible area (sign II) & 3.263 & 0.436 \\
\hline Readable area (sign II) & $7.738^{*}$ & 0.000 \\
\hline Post sign area (sign II) & $10.580^{*}$ & 0.000 \\
\hline \multicolumn{3}{|l|}{ Visible area } \\
\hline Initial speed area & 0.713 & 1.000 \\
\hline Readable area & -1.199 & 0.991 \\
\hline Post sign area & 0.274 & 1.000 \\
\hline Visible area (sign II) & 3.976 & 0.202 \\
\hline Readable area (sign II) & $8.451^{*}$ & 0.000 \\
\hline Post sign area (sign II) & $11.293^{*}$ & 0.000 \\
\hline \multicolumn{3}{|l|}{ Readable area } \\
\hline Initial speed area & 1.912 & 0.911 \\
\hline Visible area & 1.199 & 0.991 \\
\hline Post sign area & 1.472 & 0.974 \\
\hline Visible area (sign II) & $5.174^{*}$ & 0.031 \\
\hline Readable area (sign II) & $9.650^{*}$ & 0.000 \\
\hline Post sign area (sign II) & $12.492 *$ & 0.000 \\
\hline \multicolumn{3}{|l|}{ Post sign area } \\
\hline Initial speed area & 0.439 & 1.000 \\
\hline Visible area & -0.274 & 1.000 \\
\hline Readable area & -1.472 & 0.974 \\
\hline Visible area (sign II) & 3.702 & 0.280 \\
\hline Readable area (sign II) & $8.177^{*}$ & 0.000 \\
\hline Post sign area (sign II) & $11.019 *$ & 0.000 \\
\hline \multicolumn{3}{|l|}{ Visible area (sign II) } \\
\hline Initial speed area & -3.263 & 0.436 \\
\hline Visible area & -3.976 & 0.202 \\
\hline Readable area & $-5.174 *$ & 0.031 \\
\hline Post sign area & -3.702 & 0.280 \\
\hline Readable area (sign II) & 4.476 & 0.101 \\
\hline Post sign area (sign II) & $7.317^{*}$ & 0.000 \\
\hline \multicolumn{3}{|l|}{ Readable area (sign II) } \\
\hline Initial speed area & $-7.738^{*}$ & 0.000 \\
\hline Visible area & $-8.451^{*}$ & 0.000 \\
\hline Readable area & $-9.650^{*}$ & 0.000 \\
\hline Post sign area & $-8.177^{*}$ & 0.000 \\
\hline Visible area (sign II) & -4.476 & 0.101 \\
\hline Post sign area (sign II) & 2.842 & 0.607 \\
\hline Initial speed area & $-10.580^{*}$ & 0.000 \\
\hline Visible area & $-11.293^{*}$ & 0.000 \\
\hline Readable area & $-12.492 *$ & 0.000 \\
\hline Post sign area & $-11.019 *$ & 0.000 \\
\hline Visible area (sign II) & $-7.317^{*}$ & 0.000 \\
\hline Readable area (sign II) & -2.842 & 0.607 \\
\hline
\end{tabular}

*The mean difference is significant at the 0.05 level 
Table 4 Tukey's post hoc analysis-DSDS

\begin{tabular}{lcc}
\hline Phase comparison & Mean difference (I-J) & Sig. \\
\hline Initial speed area & & \\
Visible area & 2.943 & 0.408 \\
Readable area & $7.924^{*}$ & 0.007 \\
Post sign area & & 0.000 \\
Visible area & -2.943 & \\
Initial speed area & 3.221 & 0.408 \\
Readable area & $4.981^{*}$ & 0.327 \\
Post sign area & & 0.045 \\
Readable area & $-6.164^{*}$ & \\
Initial speed area & -3.221 & 0.007 \\
Visible area & 1.760 & 0.327 \\
Post sign area & & 0.790 \\
Post sign area & $-7.924^{*}$ & \\
Initial speed area & $-4.981^{*}$ & 0.000 \\
Visible area & -1.760 & 0.045 \\
Readable area & & 0.790 \\
\hline
\end{tabular}

*The mean difference is significant at the 0.05 level

Table 5 Descriptive statistics and ANOVA of all signs

\begin{tabular}{llllll}
\hline All signs & $\begin{array}{l}\text { Mean } \\
(\mathrm{mph})\end{array}$ & SD & SE & $F$ & Sig. \\
\hline $\begin{array}{l}\text { Photo enforced } \\
\quad \text { signs }\end{array}$ & 46.421 & 10.319 & 0.488 & 16.723 & $0.000^{*}$ \\
$\begin{array}{l}\text { DSDS } \\
\begin{array}{l}\text { Reduced SL 35 } \\
\quad \text { signs }\end{array}\end{array}$ & 51.899 & 11.176 & 0.693 & & \\
\hline
\end{tabular}

*Significant at $95 \%$ confidence interval

To identify the most effective sign based on reduction in overall mean speeds, an ANOVA and a Tukey's post hoc analysis were performed as given in Tables 5 and 6 , respectively. Table 5 shows that there exists a statistically significant difference in mean speeds among the three work zone signs while the post hoc analysis confirms that work zone photo enforced sign was found to be the most effective in reducing speed compared to DSDS and reduced SL 35 signs. The overall reduction in speed compared to the second-most effective sign (DSDS) is $5 \mathrm{mph}$. The mean speed trends are shown in Fig. 4.

To identify the impact of socio-demographics on changes in speed, $t$ tests were conducted. The mean speeds by gender for all signs are given in Table 7. An independent samples $t$ test was conducted to identify change in speeding behavior by gender for all signs as given in Table 8 . As Levene's statistic is $>0.05$, the $t$ test is valid. The $t$ test
Table 6 Tukey's post hoc analysis—all three signs

\begin{tabular}{lcc}
\hline Phase comparison & Mean difference (I-J) & Sig. \\
\hline Photo enforced signs & $-5.036^{*}$ & \\
DSDS & $-5.586^{*}$ & 0.000 \\
Reduced SL 35 signs & $5.036^{*}$ & 0.000 \\
DSDS & 0.000 \\
Photo enforced signs & -0.549 & 0.781 \\
Reduced SL 35 signs & $5.586^{*}$ & \\
Reduced SL 35 signs & 0.549 & 0.000 \\
Photo enforced signs & & 0.781 \\
DSDS &
\end{tabular}

shows that gender is significant only for photo enforced signs in a work zone. Female participants slow down more as compared to male participants which leads to speculation that male participants tend to take more risk than their female counterparts which is in line with past studies $[33,34]$. The $t$ tests for other socio-demographics including age, income and education were found to be insignificant and were not included in the results.

\section{Conclusions}

This study investigated the impact of work zone signs on driver speeding behavior using a medium-fidelity driving simulator. A total of 264 simulation runs were conducted involving 66 participants from different socio-demographic backgrounds. The participants drove through a work zone with two out of three lanes closed on MD-295 near Baltimore, Maryland, where the traffic was set at LOS B. In the post-simulation survey, $98 \%$ of the participants agreed that the simulation session felt very realistic. An ANOVA showed that the initial signs before the start of the work zones are only effective if they are followed up by subsequent signs, which then cause a gradual decrease in speed. The average work zone speeds in the control scenario without any signs show that the signs tested in this study are indeed effective in reducing vehicle speeds in the work zone. Speed photo enforced signs were found to be the most effective (average speed reduction of $9.4 \mathrm{mph}$ ) compared to DSDS (average speed reduction of $4 \mathrm{mph}$ ) and reduced speed limit signs (average speed reduction of $3.4 \mathrm{mph}$ ), in terms of speed reduction in a work zone. Thus, speed photo enforced signs should be widely used in work zones to influence voluntary compliance of posted speed limits by motorists, thereby allowing them more time to react to changing conditions. 


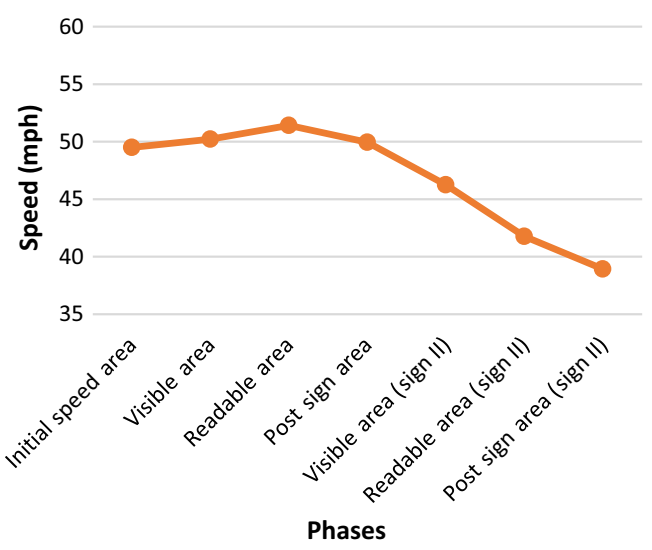

(a) Photo enforced sign speed trends

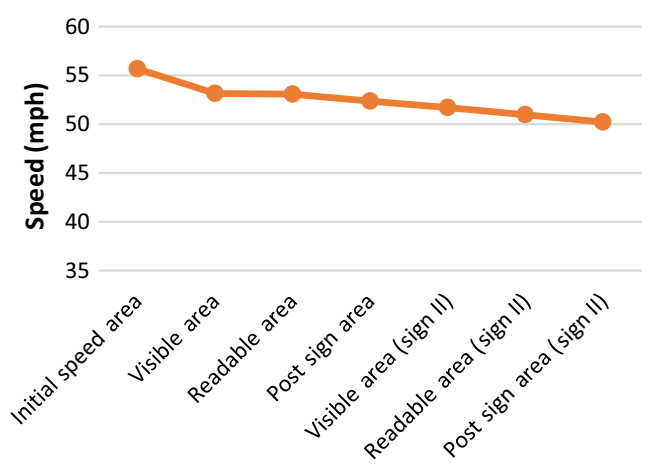

Phases

(c) Reduced SL 35 speed trends

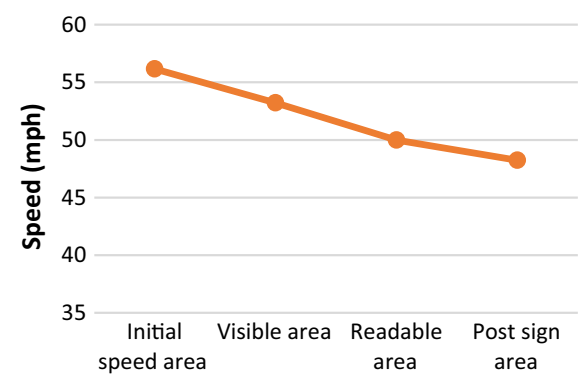

Phases

(b) DSDS speed trends

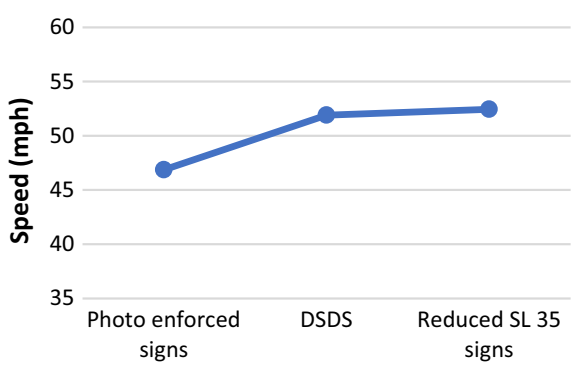

All Signs

(d) All sign speed trends

Fig. 4 Mean speed trends

Table 7 Mean speeds by gender-all signs

\begin{tabular}{llll}
\hline All signs gender & Mean speeds (mph) & SD & SE \\
\hline Photo enforced signs & & & \\
$\quad$ Male & 48.063 & 10.377 & 0.654 \\
$\quad$ Female & 45.319 & 10.061 & 0.719 \\
DSDS & & & \\
Male & 52.463 & 10.764 & 0.885 \\
Female & 51.154 & 11.706 & 1.106 \\
Reduced SL 35 signs & & & \\
Male & 52.949 & 10.230 & 0.636 \\
Female & 51.788 & 10.625 & 0.759 \\
\hline & & &
\end{tabular}

A $t$ test analysis shows that female participants tend to slow down more in the presence of speed photo enforced work zone signs. The authors propose that speed cameras along with the proper signage be installed in high-speed work zones, especially in places like tunnels where work zone crews are most vulnerable. In long-term work zones, speed photo enforced signs can be complemented with DSDS. Pilot studies should be carried out to test the effectiveness of such a combination. Future studies would involve testing a combination of speed photo enforced signs along with DSDS in a work zone and validating the results by comparing them with real-world data.

Table 8 Independent samples $t$ test-gender

\begin{tabular}{|c|c|c|c|c|c|c|}
\hline \multirow[t]{2}{*}{ All signs } & \multicolumn{2}{|c|}{ Levene's test for equality of variances } & \multicolumn{4}{|c|}{$t$ test for equality of means } \\
\hline & $F$ & Sig. & $t$ & $d f$ & Sig. (2-tailed) & Mean difference \\
\hline Photo enforced signs & 1.727 & 0.189 & 2.814 & 446 & $0.005 *$ & 2.745 \\
\hline DSDS & 0.517 & 0.473 & 0.935 & 258 & 0.351 & 1.309 \\
\hline Reduced SL 35 signs & 0.576 & 0.448 & 1.179 & 453 & 0.239 & 1.161 \\
\hline
\end{tabular}

*Significant at $95 \%$ confidence interval 
Acknowledgement The authors would like to thank the Maryland State Highway Association (SHA) for its funding support throughout the study.

Open Access This article is distributed under the terms of the Creative Commons Attribution 4.0 International License (http:// creativecommons.org/licenses/by/4.0/), which permits unrestricted use, distribution, and reproduction in any medium, provided you give appropriate credit to the original author(s) and the source, provide a link to the Creative Commons license, and indicate if changes were made.

\section{References}

1. NHTSA Work Zone Fatal Crashes and Fatalities. https://www. workzonesafety.org/crash-information/work-zone-fatal-crashesfatalities/\#national. Accessed 3 Jan 2019

2. Morris NL, Cooper JL, Ton A et al (2016) Examining the impact of ASE (automated speed enforcement) in work zones on driver attention

3. Mohan SB, Gautam P (2002) Cost of highway work zone injuries. Pract Period Struct Des Constr 7(2):68-73

4. Reyes ML, Khan SA, Initiative S (2008) Examining driver behavior in response to work zone interventions: a driving simulator study. University of Iowa, Iowa City

5. Benekohal R, Wang M-H, Chitturi M et al (2009) Speed photoradar enforcement and its effects on speed in work zones. Transp Res Rec 2096:89-97

6. Garber NJ, Patel ST (1994) Effectiveness of changeable message signs in controlling vehicle speeds in work zones. Virginia Transportation Research Council, Charlottesville

7. McAvoy DS, Center S (2011) Work zone speed reduction utilizing dynamic speed signs. Working paper, report: 01353750: University of Ohio

8. Ardeshiri A, Jeihani M (2014) A speed limit compliance model for dynamic speed display sign. J Saf Res 51:33-40

9. FHWA (2009) Manual on uniform traffic control devices for streets and highways, p 584

10. Noel E, Dudek C, Pendleton O et al (1987) Speed control through work zones: techniques evaluation and implementation guidelines. Final report

11. Bloch S (1998) Comparative study of speed reduction effects of photo-radar and speed display boards. Transp Res Rec 1640:27-36

12. Walter L, Broughton J (2011) Effectiveness of speed indicator devices: an observational study in South London. Accid Anal Prev 43(4):1355-1358

13. Crowther LR, Opiela KS (1996) Procedure for determining work zone speed limits. National Research Council, Transportation Research Board

14. Debnath AK, Blackman RA, Haworth NL (2012) A review of the effectiveness of speed control measures in roadwork zones

15. Benekohal R, Chitturi M, Hajbabaie A et al (2008) Automated speed photo enforcement effects on speeds in work zones. Transp Res Rec 2055:11-20
16. Medina J, Benekohal R, Hajbabaie A et al (2009) Downstream effects of speed photo-radar enforcement and other speed reduction treatments on work zones. Transp Res Rec 2107:24-33

17. Migletz J, Graham J, Anderson I et al (1999) Work zone speed limit procedure. Transp Res Rec 1657:24-30

18. Migletz J, Graham J, Hess B et al (1998) Effectiveness and implementability of procedures for setting work zone speed limits. Publication NCHRP project 3-41(2)

19. Bham GH, Leu MC (2018) A driving simulator study to analyze the effects of portable changeable message signs on mean speeds of drivers. J Transp Saf Secur 10(1-2):45-71

20. Venkat BK (2014) A driving simulator study to evaluate the impact of portable changeable message signs (PCMS) on driver speed characteristics

21. McCoy P, Pesti G (2002) Effectiveness of condition-responsive advisory speed messages in rural freeway work zones. Transp Res Rec 1794:11-18

22. Bham GH, Mohammadi MA (2011) Evaluation of work zone speed limits: an objective and subjective analysis of work zones in Missouri

23. Outcalt W (2009) Work zone speed control. Publication No. CDOT-2009-3. Colorado Department of Transportation, Denver

24. Banerjee S, Jeihani M, Moghaddam R (2018) Impact of mobile work zone barriers on driving behavior on arterial roads. J Traffic Logist Eng 6(2):37-42

25. Jeihani M, Banerjee S (2018) Drivers' behavior analysis under reduced visibility conditions using a driving simulator. J Traffic Logist Eng 6(2):48-52

26. Jeihani M, Banerjee S, Ahangari S, Brown DD (2018) The potential effects of composition and structure of dynamic message sign messages on driver behavior using a driving simulator (No. MD-18-SHA/MSU/4-14)

27. Jeihani M, NarooieNezhad S, Kelarestaghi KB (2017) Integration of a driving simulator and a traffic simulator case study: exploring drivers' behavior in response to variable message signs. IATSS Res 41(4):164-171

28. Harder KA, Bloomfield J, Chihak BJ (2003) The effectiveness and safety of traffic and non-traffic related messages presented on changeable message signs (CMS)

29. Kolisetty VGB, Iryo T, Asakura Y et al (2006) Effect of variable message signs on driver speed behavior on a section of expressway under adverse fog conditions - a driving simulator approach. J Adv Transp 40(1):47-74

30. Selby R (2016) Impact of dynamic message signs on driver behavior under reduced visibility conditions

31. Yan X, Wu J (2014) Effectiveness of variable message signs on driving behavior based on a driving simulation experiment. Discrete Dyn Nat Soc 2014

32. FORUM8. 3D VR \& Visual interactive simulation. http://www. forum8.com/. Accessed 25 Oct 2018

33. Gårder PE (2004) The impact of speed and other variables on pedestrian safety in Maine. Accid Anal Prev 36(4):533-542

34. Lee C, Abdel-Aty M (2005) Comprehensive analysis of vehiclepedestrian crashes at intersections in Florida. Accid Anal Prev 37(4):775-786 